\title{
The diversity and evolution of cell cycle regulation in alpha-proteobacteria: a comparative genomic analysis
}

\author{
Matteo Brilli1,2, Marco Fondi', Renato Fani', Alessio Mengoni', Lorenzo Ferri'1, Marco Bazzicalupo ${ }^{1}$ and \\ Emanuele G Biondi*1
}

\begin{abstract}
Background: In the bacterium Caulobacter crescentus, CtrA coordinates DNA replication, cell division, and polar morphogenesis and is considered the cell cycle master regulator. CtrA activity varies during cell cycle progression and is modulated by phosphorylation, proteolysis and transcriptional control. In a phosphorylated state, CtrA binds specific DNA sequences, regulates the expression of genes involved in cell cycle progression and silences the origin of replication. Although the circuitry regulating CtrA is known in molecular detail in Caulobacter, its conservation and functionality in the other alpha-proteobacteria are still poorly understood.
\end{abstract}

Results: Orthologs of Caulobacter factors involved in the regulation of CtrA were systematically scanned in genomes of alpha-proteobacteria. In particular, orthologous genes of the divL-cckA-chpT-ctrA phosphorelay, the divJ-pleC-divK twocomponent system, the $c p d R-r c d A-c / p P X$ proteolysis system, the methyltransferase $c c r M$ and transcriptional regulators $d n a A$ and gcrA were identified in representative genomes of alpha-proteobacteria. CtrA, DnaA and GcrA binding sites and $\mathrm{CcrM}$ putative methylation sites were predicted in promoter regions of all these factors and functions controlled by CtrA in all alphas were predicted.

Conclusions: The regulatory cell cycle architecture was identified in all representative alpha-proteobacteria, revealing a high diversification of circuits but also a conservation of logical features. An evolutionary model was proposed where ancient alphas already possessed all modules found in Caulobacter arranged in a variety of connections. Two schemes appeared to evolve: a complex circuit in Caulobacterales and Rhizobiales and a simpler one found in Rhodobacterales.

\section{Background}

Living cells continuously receive and process signals coming from their environment, and by integrating this information into their own internal state, are able to react with appropriate responses which coordinate each function in the cell in order to divide and produce progeny. Regulation of cell cycle progression needs to be a robust but versatile process that integrates different exogenous and endogenous signals and that guarantees fidelity and controlled progression throughout the different phases.

Bacteria have evolved different regulation systems for cell cycle coordination, probably due to different ecological and evolutionary constraints $[1,2]$. Alpha-proteobac-

\footnotetext{
*Correspondence: emanuele.biondi@unifi.it

${ }^{1}$ Department of Evolutionary Biology, University of Florence, via Romana, 17, Florence, Italy

Full list of author information is available at the end of the article
}

teria subdivision is a very heterogeneous group of bacteria and includes symbionts of plants (Rhizobia), pathogens for animals (Brucella, Rickettsia) and plants (Agrobacterium), photosynthetic bacteria (Rhodobacter) and also several genera metabolizing C1-compounds (Methylobacterium).

Together with this diversity of life styles and ecological niches, the alpha-proteobacteria subdivision is also one of the bacterial groups in which cell cycle regulation has been studied in more detail and one of its members, Caulobacter crescentus, has recently become a model organism in these studies [3-6]. In this organism each cell division is asymmetric-producing two functionally and morphologically different cells, the replicating "stalked" cell type and the vegetative "swarmer" type. After each initiation of DNA replication, the replication fork is kept 
blocked so that the Caulobacter cell cycle can follow a pattern of once-and-only-once replication per division (G1, S, and G2 phases are temporally distinguished).

Many factors are known to regulate cell cycle progression and most of them are members of the family of twocomponent signal transduction proteins, comprised of histidine kinases and their response regulator substrates [6]. Among those proteins CtrA is the master regulator of the Caulobacter cell cycle, an essential response regulator whose activity as a transcription factor varies as a function of the cell cycle [7-9]. CtrA controls various functions during cell cycle progression by activating or repressing gene expression. CtrA also blocks the initiation of DNA replication through binding of the replication origin [7]. Among genes regulated by CtrA we can find those involved in cell division (ftsZ, ftsA, fts $Q$ and $f t s(W)$, the protease encoding gene $\operatorname{clp} P$ which is essential in Caulobacter, the DNA methylase gene ccrM, flagellar biogenesis genes, stalk biogenesis regulatory genes, pili biogenesis genes such as pilA, and chemotaxis genes [1015].

CtrA activity and stability varies during the cell cycle. Oscillation of CtrA levels, peaking at the predivisional stage before cell division, is achieved by different mechanisms: transcription, proteolysis and phosphorylation control as discussed in detail below.

DnaA and GcrA, and the DNA methyltransferase CcrM are involved in controlling ctrA transcription $[11,16]$. DnaA is a key element in cell cycle regulation because it is required for the initiation of DNA replication; it also controls the transcription of about 40 genes involved in nucleotide biogenesis, cell division, and polar morphogenesis $[17,18]$, and it activates the transcription of the $g c r A$ gene [19]. GcrA controls the transcription of ctrA and genes involved in DNA metabolism and chromosome segregation, including those encoding DNA gyrase, DNA helicase, DNA primase, and DNA polymerase III [19]. As a consequence of this genetic circuit, CtrA accumulates out-of-phase with GcrA [19]. The transcriptional loop of $c t r A$ is closed by CcrM. CtrA activates the transcription of $c c r M$, which encodes for a DNA methyltransferase whose abundance is cell cycle dependent. CcrM is able to activate dnaA promoter region through methylation, closing the positive feedback composed by CtrA, DnaA and GcrA.

A second essential regulatory control on CtrA is carried out by phosphorylation. In fact, CtrA must be phosphorylated to bind DNA and its phosphorylation depends on cell cycle progression. An essential phosphorelay, composed of the hybrid histidine kinase CckA and the histidine phosphotransferase ChpT, is responsible for CtrA phosphorylation $[20,21]$.

DivK, which is a response regulator, plays an essential role as a positive regulator of cell cycle progression because when phosphorylated, it indirectly inactivates CtrA and thus promotes DNA replication. Two histidine kinases are known to interact with DivK: PleC and DivJ [22-25]. Bacterial histidine kinases can have alternatively both kinase and phosphatase activities and these opposite activities are modulated by conformational changes of the protein [26]. A null Caulobacter pleC mutant produces almost symmetric cells at the division and displays abnormal polar development. The DivJ histidine kinase plays a role in controlling the length and location of the stalk and cell division. PleC and DivJ are considered the principal phosphatase and kinase, respectively, of DivK and they are in opposite locations during cell cycle progression $[27,28]$. Divj activity is also positively controlled by the TacA/SpmX pathway, which is transcriptionally activated by CtrA $[10,29]$.

ChpT also transfers the phosphate to a second response regulator named $\mathrm{CpdR}$, which, together with $\operatorname{RcdA}$, is a factor involved in CtrA proteolysis mediated by ClpPClpX protease [30-32]. CtrA is degraded at the stalked pole at the G1/S transition when the origin of replication needs to be cleared and also in the stalked compartment, where initiation of DNA replication occurs immediately after cell division [33,34].

All these regulations are schematized in Figure 1 where we illustrate the multilevel regulation of the Caulobacter cell cycle. Two main oscillators are working during cell cycle progression: (i) the transcriptional and epigenetic circuit (CtrA-DnaA-GcrA-CcrM); (ii) the phosphorylation/proteolysis and transcription circuit (CckA-CtrADivK). The latter also involves coordination of CtrA proteolysis and cell division through regulation of DivK activity.

Several of these regulatory mechanisms are at least partially redundant, and it has been demonstrated that only phosphorylation of CtrA is indispensable during cell cycle progression; in fact, cell cycle regulated transcription of ctrA can be substituted by constitutive transcription [20] and proteolysis can also be removed.

It has recently been demonstrated that asymmetric division also takes place in Agrobacterium tumefaciens, Sinorhizobium meliloti and Brucella abortus [35], indicating that at least some of the features governing cell cycle progression in Caulobacter might also be present in other species. For example, in Rhodobacter capsulatus, CtrA and CckA are not essential but are required for the expression of the GTA, a system for genetic exchanges [36,37]. CtrA in Brucella controls cellular events similar to those controlled by CtrA in Caulobacter, but through a direct effect on different targets [38]. Moreover CtrA from Caulobacter is able to bind the B. abortus ccrM promoter in vitro [39] and CtrA from B. abortus has been shown to bind promoters of $c c r M$, pleC, rpoD, ftsE and $\min C$ but not $\operatorname{divK}, \mathrm{fts} Z$ or the origin of replication, that 


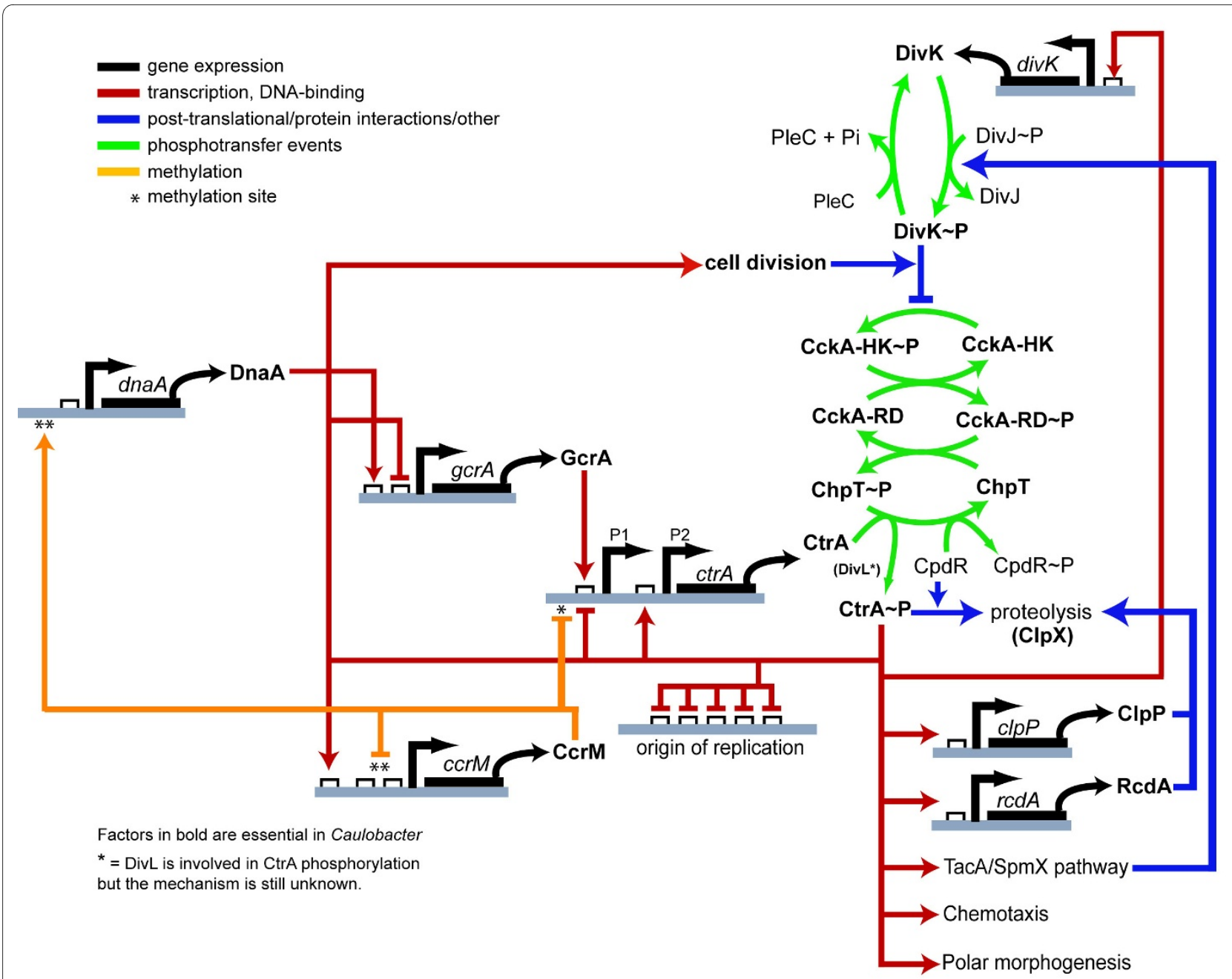

Figure 1 Cell cycle regulation in Caulobacter. Scheme of cell cycle regulation in Caulobacter with all factors analyzed in this paper. See the "introduction" for details.

are known CtrA targets in Caulobacter [38]. In Silicibacter pomeroy three known mutants affect the motility, two of which are $c c k A, c t r A[40]$.

ccrM is essential and cell cycle regulated in A. tumefaciens [41], as observed in B. abortus, where $c c r M$ is also essential and its overexpression impairs proper intracellular replication in murine macrophages [39], revealing a link between cell cycle and pathogenetic activity.

We hereby undertook a comparative and integrative analysis of factors controlling cell cycle regulation, and the regulatory connections existing between them, looking for orthologous genes of factors that are involved in controlling CtrA, the master regulator, in C. crescentus. We also analyzed CtrA-regulated functions in 37 representative genomes of alphas. At the same time, binding sites of DnaA, GcrA and the presence of putative methylation sites of CcrM were also suggested through bioinformatic analysis. All this information was used to reconstruct the architecture of CtrA regulation throughout the phylogenetic tree of alpha-proteobacteria to reveal evolutionary trends and insights into this complex regulation.

\section{Results}

\section{Evolutionary scheme of alpha-proteobacteria}

To construct a robust phylogeny of alpha-proteobacteria, a dataset of eight universal proteins from the Ribosomal Database Project was downloaded. Proteins were aligned separately and then the alignments were concatenated, resulting in an alignment of 5056 amino acids that has been used to construct a Neighbor-Joining tree (see Methods) (Figure 2). A comparison with previous phylogenetic trees of alpha-proteobacteria [42] suggested that the tree root lies in the branch connecting the Rickettsiales to the other alpha-proteobacteria. Unlike the work of Gupta and Mok (2007), our tree shows Sphin- 


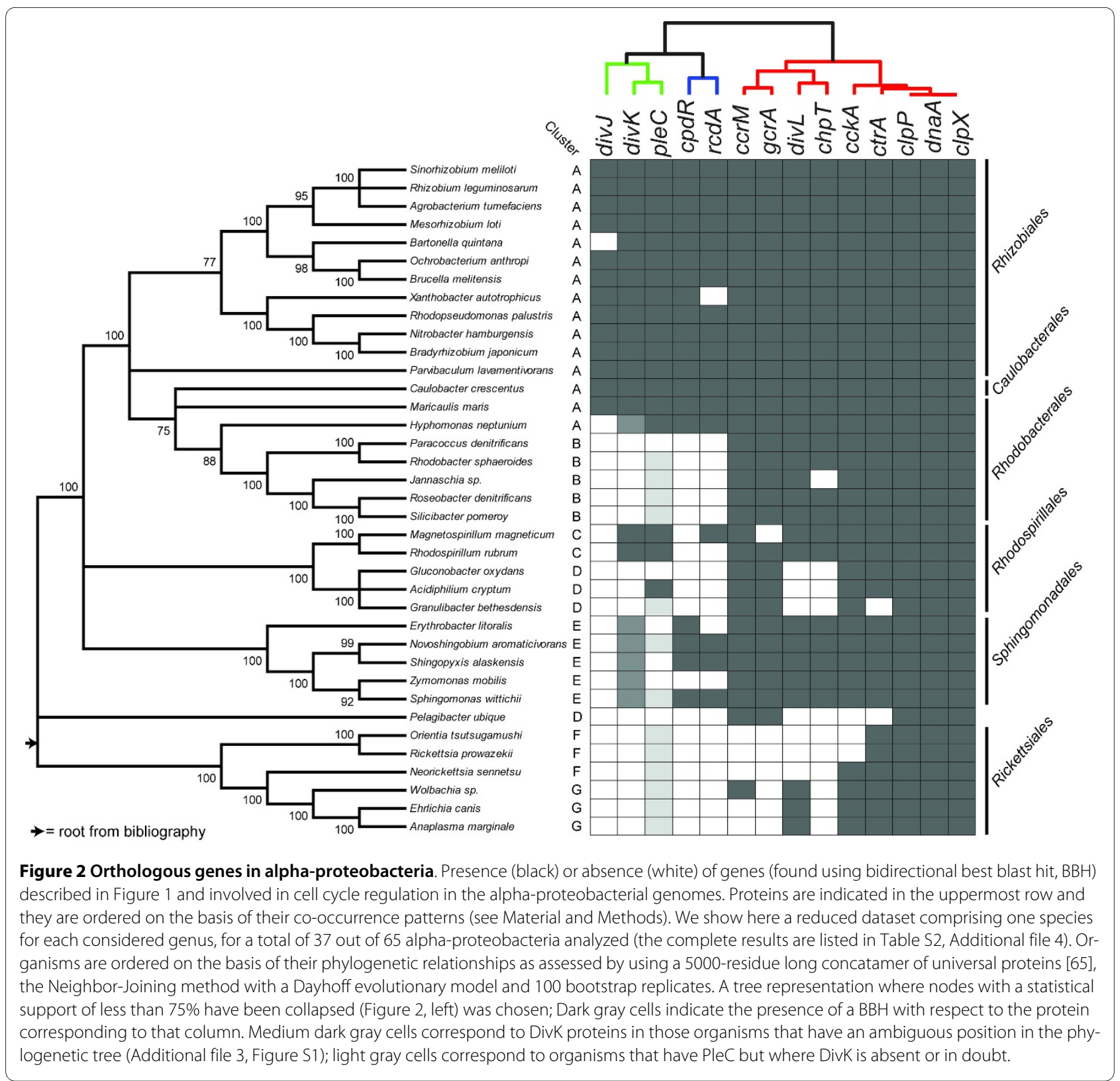

gomonadales branching off after the Rickettsiales and followed by the branching of Rhodospirillales, whereas in other works the latter branched first.

\section{Clusterization of alpha-proteobacteria based on the orthology of cell cycle genes}

By using the bidirectional best hit $(\mathrm{BBH})$ approach (see Material and Methods section) on 65 available genomes of alpha-proteobacteria (Additional file 1, Table S1; legends of additional Figures and Tables are in Additional file 2) we obtained a list of genes that are orthologous to the 14 genes involved in the Caulobacter cell cycle, and the results are reported in Figure 2 (see also Additional file 3, Figure S1 and Additional file 4, Table S2).

ClpX, ClpP and DnaA are present in all alphas studied, but surprisingly all other proteins analyzed can be absent in several alphas. Transcription factors, GcrA and CtrA, the DNA methyl-transferase CcrM and the hybrid histidine kinase CckA are present in most of the alpha-proteobacteria. Other modules, such as those of the DivJPleC-DivK two-component system, are present only in clusters $\mathrm{A}$ and $\mathrm{C}$ of alpha-proteobacteria.

Genes with similar phylogenetic profiles (genes cooccurring in different genomes) are often functionally related [43] justifying the use of our profiles to investigate possible functional relationships; the dendrogram obtained describes how similar the profiles of different 
genes are (see Methods and upper part of Figure 2) and it confirms the functional association between divJ, pleC and $\operatorname{div} K$ (encoding the two component system negatively regulating CckA activity), and between $c p d R$ and $r c d A$, whose products are involved in CtrA proteolysis. Weaker and possibly new functional associations concern the

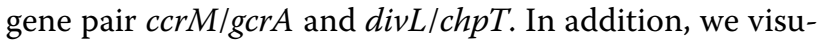
ally inspected the phylogenetic profiles of these genes between organisms, identifying seven groups (from A to G, see Figure 2). This classification, based on orthology, will be used as a reference in the following sections.

Cluster A includes Rhizobiales, Caulobacterales and several Rhodobacterales, and is composed of the largest number of sequenced genomes; this cluster is characterized by a nearly identical conservation of factors found in Caulobacter. Although similarities are evident in this cluster, a deeper analysis revealed substantial differences that will be discussed in the next sections.

Cluster B, including other Rhodobacterales, shows a substantial difference compared to cluster A; in fact, both DivJ-PleC-DivK and RcdA-CpdR systems are missing.

Magnetospirillum and Rhodospirillum, which are closely related, are the two members of cluster C. This cluster is characterized by the presence of the PleC-DivK system since DivJ is missing and by an almost complete loss of the CpdR-RcdA system although Magnetospirillum possesses an $r c d A$ orthologous gene.

Clusters D (Rhodospirillales) and F (part of Rickettsiales), even though they are separated in the tree reported in Figure 2, share common features: i. members of the CckA-ChpT-CtrA phosphorelay are missing at different degrees (Granulibacter and Pelagibacter do not show even $c$ trA orthologs); ii. Divj-PleC-DivK and RcdA-CpdR systems are missing. Despite these similarities, the two groups diverge for the presence of CcrM and GcrA in group D.

Organisms belonging to cluster E (Sphingomonadales) show conservation of the phosphorelay CckA-ChpT$\mathrm{CtrA}$ and also often possess factors required for the temporally and spatially regulated proteolysis of CtrA, such as $\mathrm{CpdR}$ and RcdA. However cluster $\mathrm{E}$ is characterized by degeneration or a complete loss of the DivJ-PleC-DivK regulation system. DivK or PleC orthologs can be found in several organisms of this subgroup although their phylogeny often significantly deviate from the phylogenetic tree of housekeeping genes (Additional file 5, Figure S2).

Finally, group G (remaining Rickettsiales), composed mainly of pathogens, has few of the factors involved in Caulobacter cell cycle progression regulation. It is however interesting to find a CckA-CtrA system whereas ChpT orthologs cannot be found using the $\mathrm{BBH}$ approach.

\section{CtrA regulon in alpha-proteobacteria}

The regulatory circuit that controls cell cycle progression in Caulobacter is also composed of crucial transcriptional connections, such as CtrA control on divK and the CtrADnaA-GcrA-CcrM circuit. This transcriptional regulation level is discussed in this section and the following. In particular, results obtained for the prediction of the CtrA regulon in alpha-proteobacteria are described here.

Laub and collaborators $[13,44]$ were able to identify a set of genes plausibly constituting the CtrA regulon in Caulobacter by combining varying evidence: 116 genes were identified through chromatin immunoprecipitation using phosphorylated CtrA; 88 genes were identified as CtrA-dependent for normal expression levels, and 69 as cell cycle regulated in a transcriptome analysis encompassing one complete cell cycle round. The 54 genes within the overlap of all three data sets were identified as members of the CtrA cell cycle regulon, and were used here to build the position weight matrix (PWM) of CtrA binding sites. Upstream sequences of these 54 genes were retrieved and used to find enriched sequence motifs using AlignAce [45]. The PWM obtained (Additional file 6, Table S3) corresponds to a 16-mer containing the known CtrA binding motif and was used in a sliding window approach on a non-redundant subset of the genomes used in this work. An output was obtained where genes in a given genome have a score based on the presence of CtrA sequence motifs in the region comprised of 100 nucleotides within the coding sequence to 400 nucleotides upstream of the start codon (see Methods for details).

Is the CtrA PWM, based on Caulobacter data, valid for all the alphas analyzed here? One might speculate that if a ctrA gene taken from an alpha is able to complement deletion in Caulobacter, the PWM built on Caulobacter ctrA-controlled genes would also be valid for the bacterium where the complementing $\operatorname{ctr} A$ comes from. It has been shown that the $c t r A$ gene from $R$. prowazekii, named $c z c R$, is able to complement the deletion of $c t r A$ in Caulobacter, confirming that the functionality (that is, the binding site) is conserved between Rickettsia and Caulobacter [46]. Moreover other ctrA genes from species taxonomically closer to Caulobacter, such as S. meliloti, are able to complement the ctrA deletion in Caulobacter (Biondi, unpublished data). Considering the phylogeny of alphas and positions in the tree of $R$. prowazekii and $S$. meliloti, it is reasonable to consider that the CtrA binding site might be substantially conserved across the alphas.

Two kinds of results from this analysis are shown here: (i) CtrA target genes belonging to our starting dataset of cell cycle related genes (Figure 3) and (ii) enrichment of COG (clusters of orthologous groups of proteins) categories of genome-wide CtrA regulons for each genome analyzed (Figure 4). 


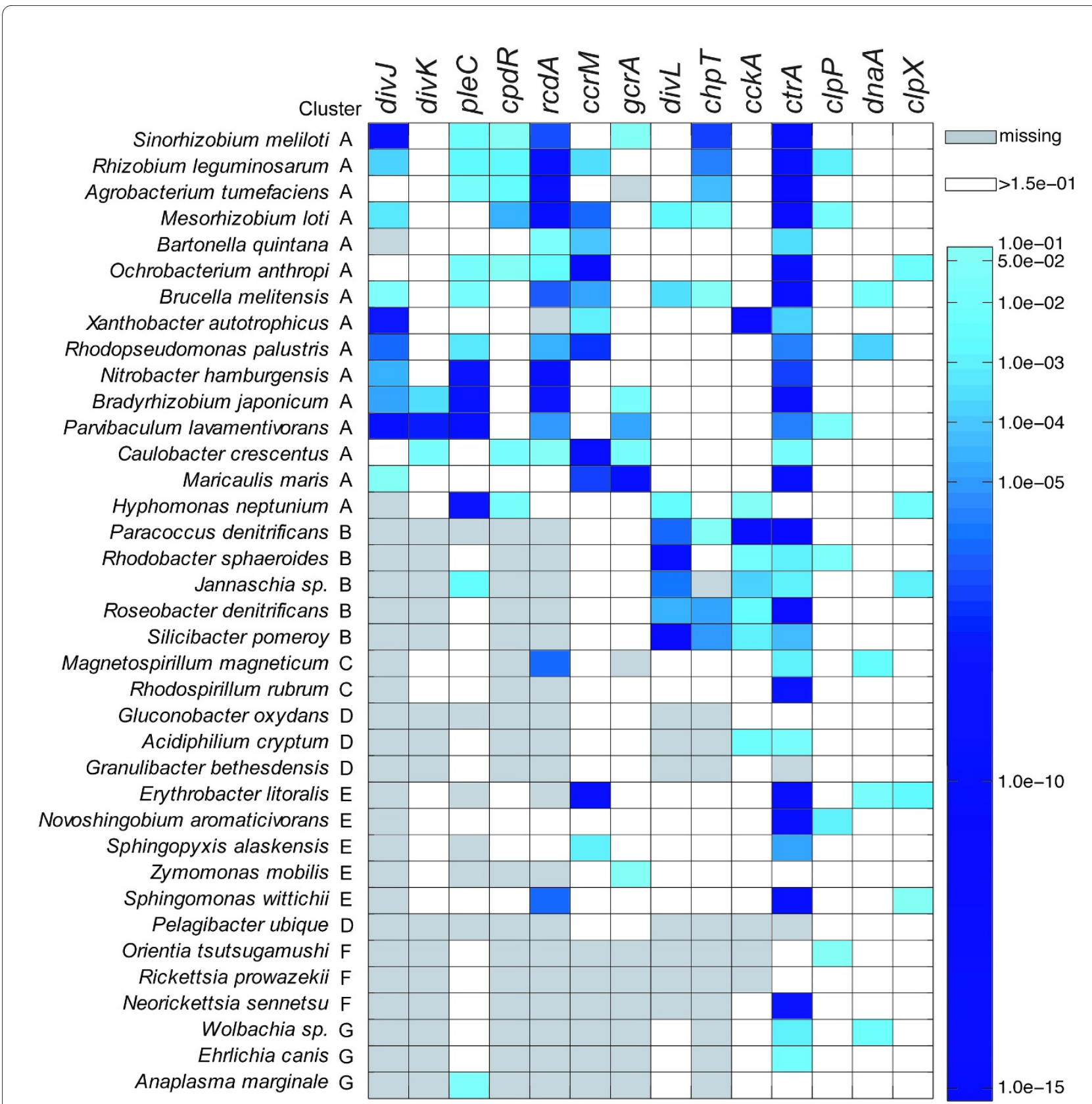

Figure 3 Transcriptional control of CtrA on cell cycle genes. Transcriptional control of CtrA on factors involved in regulation of cell cycle progression (see also Additional file 7, Table S4). Color bar represents p-values of the Z-score transformation of motif scores (Methods section for details).

In Figure 3 (see also Additional file 7, Table S4) we show the p-values for the presence of CtrA binding sites upstream of analyzed genes. CtrA controls the transcription of several genes involved in regulation of cell cycle progression, including itself in most of the alphas analyzed (86\%). Moreover, the number of genes controlled by $\mathrm{CtrA}$ is maximal in cluster A. In this cluster CtrA controls at least one gene of each of the following systems: DivJDivK-PleC, CpdR-RcdA-ClpPX and GcrA-DnaA-CcrM. Several genes showed evolutionary conservation of CtrA control among members of cluster A, such as DivJ, RcdA and CcrM. The second important result arising from the analysis shown in Figure 3 was that in Cluster B, where the DivJ-PleC-DivK system is missing, CtrA controls both CckA and DivL.

Each genome-wide regulon of $\mathrm{CtrA}$ was defined as the list of genes with a Z-score (see Methods) $\geq 2$ (corresponding to a p-value of ca. 0.023) in an organism. In Figure 4 (see also Additional file 8, Table S5), predicted regulons of CtrA were analyzed for functional enrichment (percentage of genes in a COG category controlled by $\mathrm{CtrA}$ ) in genes belonging to functional categories. 


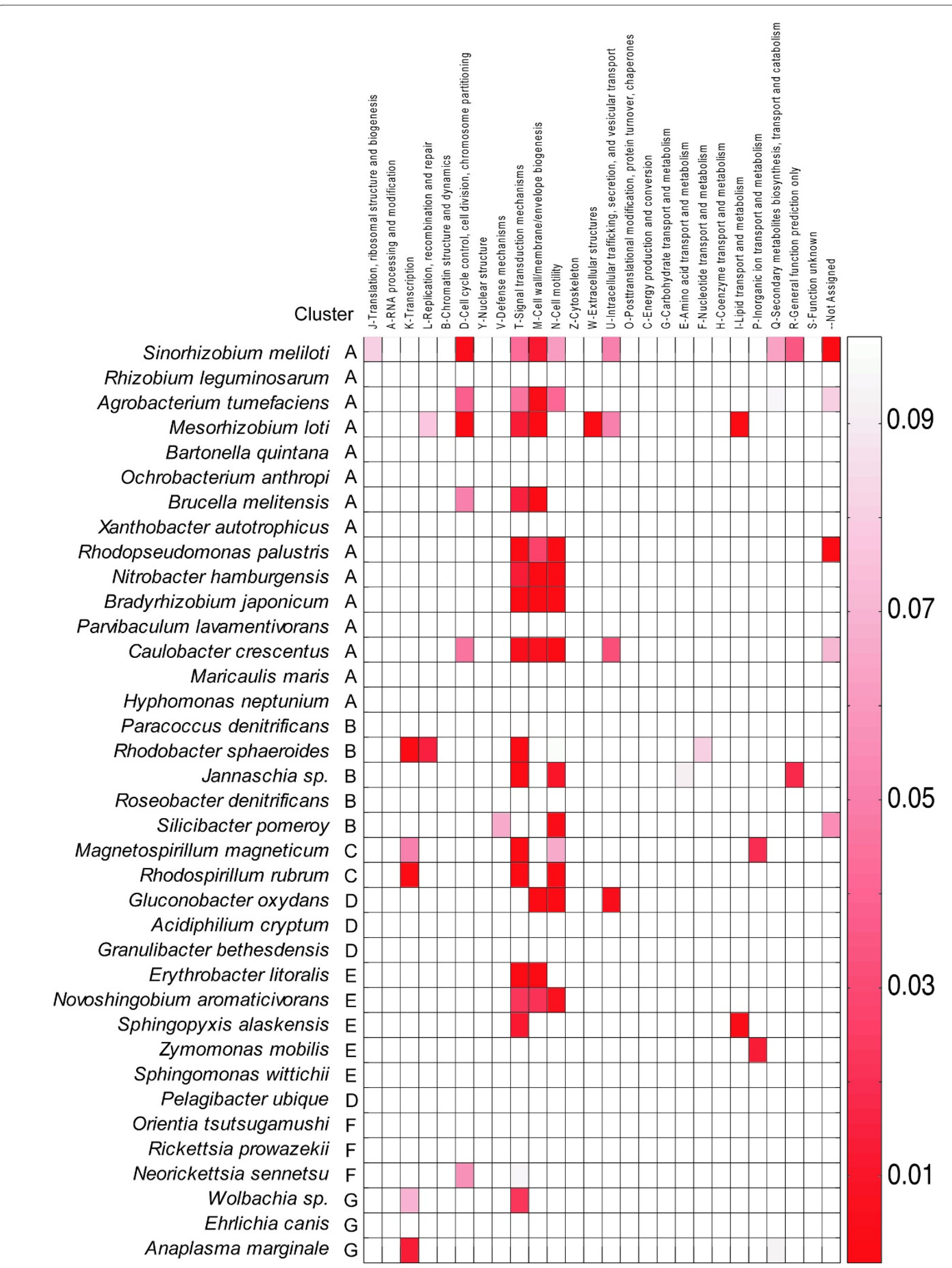

Figure 4 Functions controlled by CtrA among alpha-proteobacteria. Enrichment of genes controlled by CtrA in COG categories (see also Additional file 8, Table S5). The scale corresponds to the $\mathrm{p}$-value of the functional enrichment calculated as described in the Material and Methods. The $\mathrm{p}$ value is inversely correlated to the strength of the functional enrichment of each regulon, i.e. a lower p-value indicates stronger enrichment. 
Most enriched categories were Signal transduction mechanisms (enriched in 15 organisms), Cell wall/membrane/ envelope biogenesis (enriched in 10 organisms) and Cell motility (enriched in 9 organisms), while cell cycle functions were enriched in six species, belonging to cluster A, including Caulobacter and Neorickettsia sennetsu of cluster F. These results confirmed experimental data on the functions controlled by CtrA (see Background section) in Caulobacter suggesting that (i) the analysis of the regulon is able to capture good candidates of CtrA targets and (ii) the control of CtrA over these functions is at least partially evolutionarily conserved.

\section{CtrA-DnaA-GcrA-CcrM connections}

In Caulobacter, transcriptional regulation of ctrA is based on a positive feedback loop that includes CtrA itself, GcrA, DnaA and CcrM.

As reported in Figure S3 (Additional file 9) (see also Additional file 7, Table S4) the presence of CcrM methylation sites upstream of cell cycle related genes was assessed (see Methods section). For this analysis we used the consensus methylation site of CcrM, i.e. GANTC [47]. As reported elsewhere [39,48], methylation by CcrM is conserved among alphas and $\mathrm{ccrM}$ genes from $\mathrm{Cau}$ lobacter and $S$. meliloti which are able to cross-complement deletions, suggesting that the methylation site might be conserved. In a homogeneous background of DNA, the expected frequency of this sequence is $4 / 1024$ nucleotides, i.e. two occurrences in the $500 \mathrm{bp}$ long window that was used to define the promoter region for motif finding. The average number of occurrences found ranged from 0.4 to 1.5 methylation sites per promoter (defined as the $500 \mathrm{bp}$ from the first $100 \mathrm{bp}$ of a gene to $400 \mathrm{bp}$ upstream of the translation start site), according to non-uniform distributions of nucleotides in these genomes. Although some genes possess more predicted methylation sites in their promoter region, it is not possible to derive a possible control of methylation in their expression.

The consensus site of DnaA is very conserved among bacteria, in fact, DnaA experimental binding sites in $E$. coli and $B$. subtilis differ only by a nucleotide $[49,50]$ which corresponds to that found in Caulobacter, supporting the conclusion that its binding site is conserved across alphas. Promoter regions of orthologs in all alphas were also scanned using the DnaA matrix based on 15 known DnaA motifs in Caulobacter (see Methods section) and results are shown in Figure 5 (see also Additional file 7, Table S4). Again, is the DnaA binding site conserved across the alpha proteobacteria? Following this analysis, the promoter sequence of $g c r A$ does not bear a significant DnaA motif in Caulobacter, while elsewhere the control of DnaA on the $g c r A$ promoter has been proposed [51]. It is worth noting that a predicted DnaA motif is present upstream of the $g c r A$ gene from two closely related species Hyphomonas neptunium and Maricaulis maris. Moreover, in Caulobacter, the presence of DnaA binding sites was observed upstream of $\operatorname{divJ}$ and $c c k A$, targets that are not confirmed by previous experimental analysis. As in the case of GcrA, the absence of conservation of putative binding sites at the taxonomic level was observed, with the only exception being the suggested DnaA control on CtrA in several species. This might be caused by the low specificity of the DnaA and GcrA matrices used for motif finding, but may also suggest that in some alpha-proteobacteria the DnaA $>$ GcrA $->$ CtrA circuit may be simplified by excluding GcrA. The control of DnaA on CcrM is also interesting because it mainly concerns those organisms where $c c r M$ lacks CtrA binding sites. The opposite is also true: $c c r M$ is very often preceded by CtrA binding sites in Rhizobia and it lacks DnaA motifs. This questions the existence of a DnaA->CcrM$>$ CtrA circuit in Rhizobia and suggests an at least partial decoupling of CtrA activity (modulated through control of the divJ-pleC-divK system, which acts on the phosphorelay) from DNA replication triggered by DnaA. Otherwise, the absence of CtrA binding sites upstream of DnaA might suggest the existence of other not yet identified regulators, which may connect CtrA and DnaA in these organisms.

Although GcrA is considered a DNA binding protein activating transcription, no experimental evidence has ever been proposed to demonstrate this behavior [19] However it is still possible that DNA sequences are associated with the presence of this factor. GcrA putative binding sites were searched for in promoter regions of genes encoding factors involved in cell cycle regulation using a strategy similar to the one followed to predict CtrA regulons (see Methods section). In C. crescentus, as reported in Figure 5 (see also Additional file 7, Table S4), the existence of a putative GcrA binding motif (Additional file 10, Figure S4) upstream of ctrA and also the presence of such motifs upstream of divJ was confirmed. Concerning the other species, patterns of occurrence did not respect phylogenetic relationships. The only gene for which most of the organisms seem to possess a GcrA binding site is $c l p X$.

\section{Verification of binding site prediction}

The prediction of CtrA and DnaA binding sites across alphas is based on Caulobacter data and we have already discussed how, from previous studies, it is possible to hypothesize that CtrA and DnaA (and also CcrM) binding sites are conserved across the alpha-proteobacteria. However our prediction ability might be accurate only for bacteria closely related to Caulobacter, but, going farther, this confidence could decrease. To evaluate this bias in binding site prediction, we counted the number of genes 


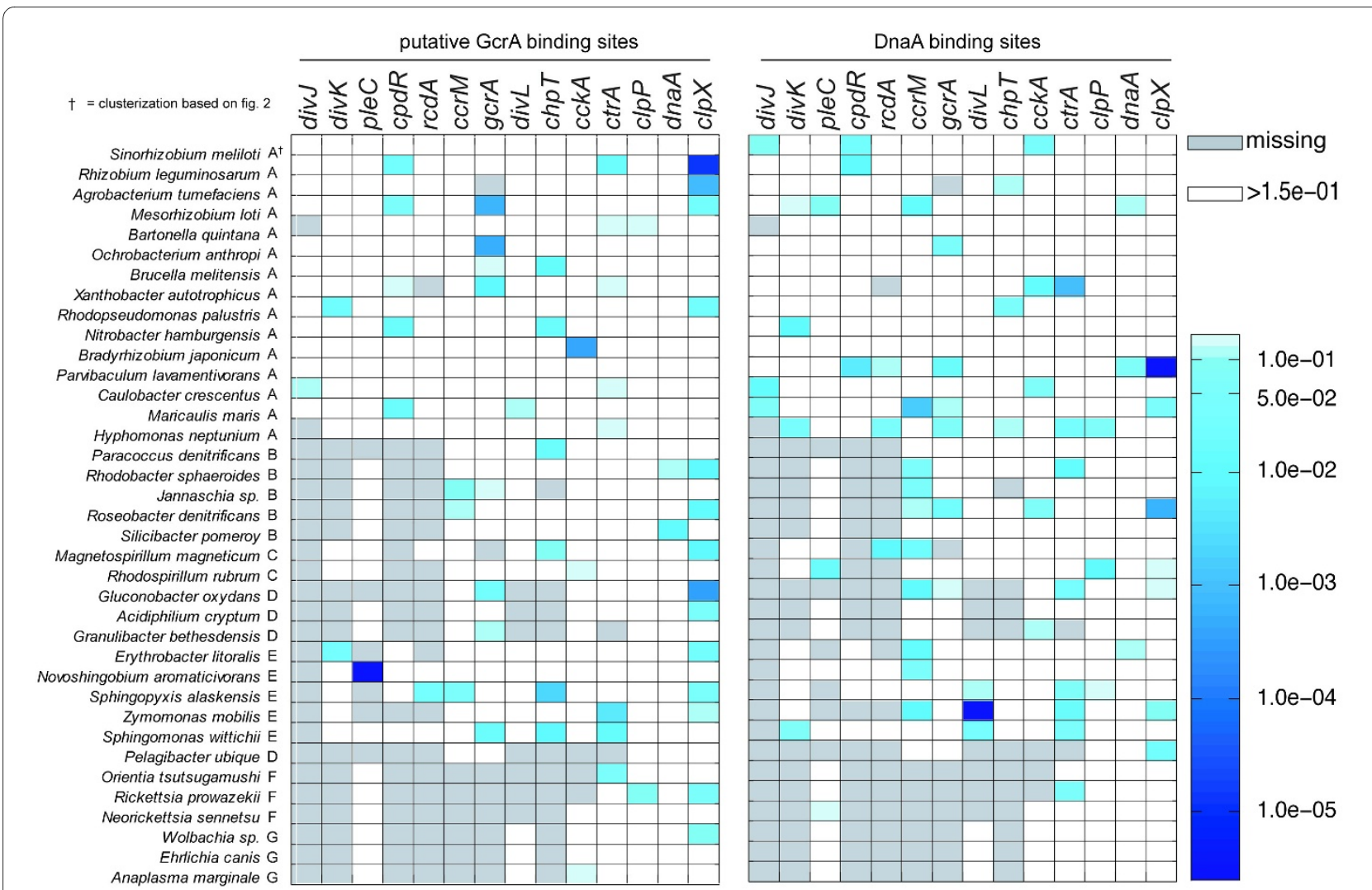

Figure 5 Transcriptional control of GcrA and DnaA on cell cycle genes. GcrA and DnaA binding sites on factors involved in cell cycle regulation among alpha-proteobacteria (see also Additional file 7, Table S4).

in each genome putatively controlled by CtrA and DnaA, normalized for the genome size. We found (Figure 6A) that the number of predicted genes is fairly constant and depends only on the genome size (or number of genes), suggesting that our prediction confidence is not biased by the phylogenetic distance. This result also explains the success of the complementations of ctrA deletion in Caulobacter by orthologs from other alpha proteobacteria, as discussed in the previous sections $[46,52]$. We also evaluated whether the presence of CtrA and DnaA predicted genes depended on the presence of CtrA and DnaA themselves in the genomes or if it was an artifact of bioinformatic analysis. We therefore plotted the fraction of genes controlled by CtrA and DnaA at small p-values in three alpha proteobacteria possessing CtrA and DnaA and in E. coli and B. subtilis, which possess only DnaA (Figure 6B). From this analysis it is evident that, at lower p-values, only organisms with CtrA keep a consistent fraction of genes controlled by CtrA, while for DnaA, which is present and active in all, every organism maintains a similar fraction of putatively controlled genes-even at lower p-values.

CtrA binding site consensus has been previously tested experimentally in R. prowazekii, S. meliloti and B. abor- tus, besides Caulobacter [38,46,52]. Here, we compared the experimental consensus sequences with our bioinformatic PWM (Figure 6C), and our prediction coincides with experimentally identified sequences. Our PMW corresponds also with a CtrA PMW previously found [53].

The DnaA binding site has been studied in very diverse bacteria such as Gram-negative Escherichia coli and Gram-positive Bacillus subtilis [49,50]. The DnaA binding site in these two species differs because of one nucleotide, suggesting that the binding site should also be very conserved in alpha proteobacteria. We compared the predicted PWM for DnaA based on C. crescentus with experimental DnaA binding sites in E. coli, B. subtilis and $S$. meliloti (Figure 6C) [49,50,54]. Our prediction, based on nucleotide sequences that bind DnaA in Caulobacter, corresponds to binding sites experimentally found in other bacteria.

This verification was possible only for DnaA and CtrA, while GcrA has been studied only in C. crescentus and experimental data are available only in this organism. It has not been clarified whether GcrA binds DNA directly or through an unknown factor X [19]. Therefore, since the knowledge on GcrA is still preliminary and experimental work still needs to be done, we limited the experi- 


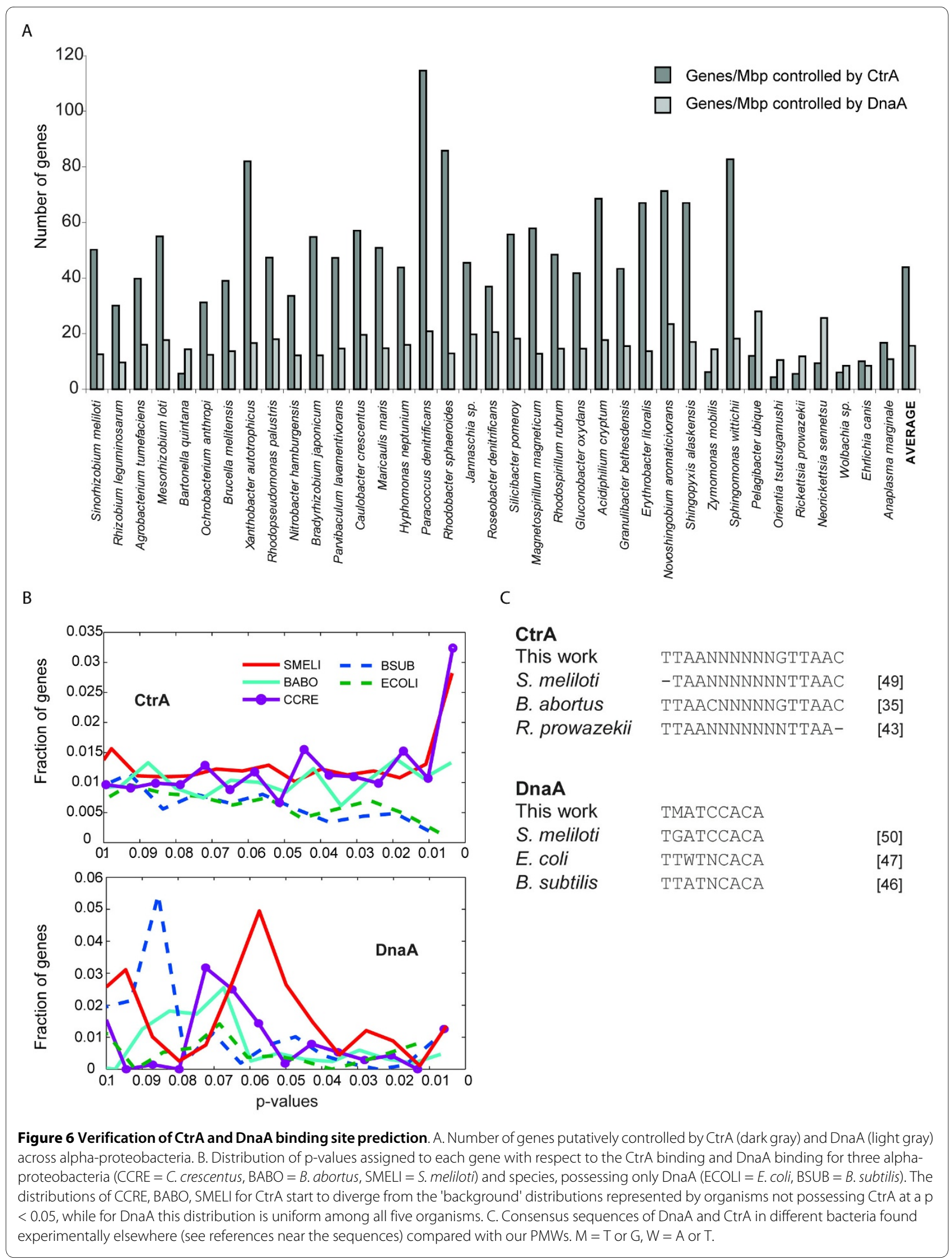


mental validation to $\mathrm{Ctr} A$ and $\mathrm{DnaA}$, for which data are available. It should be noted, however, that both DnaA and CtrA experimental verifications revealed that our method is accurate and reliable.

\section{Reconstruction of regulatory circuits}

Based on data of Figures 2, 3, and 5, we reconstructed the architecture of the seven clusters (A to G) found in the $\mathrm{BBH}$ analysis; as discussed below, only four clusters revealed a defined architecture as illustrated in Figure 7. Models of CtrA regulation are shown in the clusters (clusters A, B, C and E) where interactions between factors were found. This modeling is essential in order to underline differences and conservation of several features of cell cycle regulation in alpha proteobacteria.

Cluster A (Caulobacterales, Rhizobiales and several Rhodobacterales) contains the larger number of genomes analyzed here and the organization of cell cycle genes resembles that observed in Caulobacter (see bottom part of Figure 7 for details), i.e. it includes the phosphorelay CckA-ChpT-CtrA/CpdR and also the proteolysis machinery composed by the ubiquitous ClpPX protease, CpdR and RcdA, which is however absent in Xanthobacter. The DivJ-PleC-DivK system is conserved and corresponding genes are controlled by $\mathrm{Ctr} A$ in all members of the cluster. CcrM also controls CtrA and GcrA.

Rhizobiales are different from bacteria similar to Caulobacter (B. japonicum, P. lavamentivorans and M. maris) due to the absence of the CtrA control on GcrA which is present only in the Caulobacter-like.

In cluster E, the CckA-ChpT-CtrA phosphorelay is present with the second branch also leading to phosphorylation of $\mathrm{CpdR}$ that, together with RcdA, are thought to be involved in controlling CtrA proteolysis. DivK is absent in this cluster although a divK-like gene has been found although it has an anomalous phylogeny (Additional file 3, Figure S1). In most members of cluster B, CtrA controls its own promoter, other genes involved in cell division and chromosome partitioning as well as ccrM.

In cluster $\mathrm{B}$, the CckA-ChpT-CtrA regulon is isolated from GcrA, CcrM and DnaA while CtrA controls itself as well as two factors involved in its phosphorylation, CckA and DivL.

In this group, DivL lacks the kinase domain that is usually present only when DivK is also present (data not shown, based on SMART database). In fact, DivK is absent in cluster B together with its kinase/phosphatase. The fact that CtrA controls its kinase creates theoretically a feedback.

In cluster $\mathrm{C}$, the CckA-ChpT-CtrA phosphorelay is present while CpdR is absent. Also CtrA is not connected with DnaA and CcrM and finally DnaA has binding sites on $c c r M$ and $\operatorname{divL}$. Connections between DnaA/CcrM and CtrA seem to be achieved by the PleC-DivK two- component system. No clear positive or negative transcriptional feedbacks of CtrA on other cell cycle factors are present.

Cluster D contains the two cases among alphas, Granulibacter and Pelagibacter, where a ctrA ortholog has not been found. The phosphorelay, even in organisms of cluster $\mathrm{E}$ that have $\mathrm{Ctr} A$, is degenerated; although $c c k A$ is present, no orthologous gene of $\operatorname{ch} p T$ has been found. There is no explanation for the presence of CckA in organisms with no CtrA. Since histidine phosphotransferases are difficult to annotate $[10,20]$, it is possible that other phosphotransferases substitute for ChpT in those organisms containing both $\mathrm{Ctr} A$ and CckA, as proposed here for cluster $\mathrm{G}$.

\section{Discussion}

The cell cycle 'engine' (controlling DNA replication, cell division, morphogenesis of polar structures) is an essential machine in every living organism, and a major goal of molecular cell biologists is to uncover how this engine works in every organism. We have performed a bioinformatic analysis of cell cycle control genes in the alpha proteobacteria, taking cues from the well-characterized control system in Caulobacter (see Background section for details).

The procedure used here is able to detect only loss of regulatory points with respect to Caulobacter. However, the knowledge of conservations and variations from the Caulobacter scheme can be extremely useful for future studies of the cell cycle network in all alpha-proteobacteria.

\section{Conservation and variability of the CtrA regulatory system}

CtrA regulation is thought to play an essential role in most alpha-proteobacteria cell cycle progression while its activity varies during each cell cycle in response to several levels of control in Caulobacter. As illustrated in Figure 7, many regulators are connected with $\mathrm{Ctr} A$, via epigenetic, transcriptional and post-translational mechanisms in most of the clusters. The reconstructed architecture within alpha-proteobacteria of regulatory pathways that are involved in controlling CtrA activity is however surprisingly variable, suggesting diverse evolutionary pathways in each cluster.

CtrA, even if associated with variable regulatory circuits within alphas, shows conservation of the control of certain functions (Figure 4) such as cell division, motility and signal transduction, especially in cluster A where $\mathrm{CtrA}$ is, in fact, the master regulator of cell cycle. The ability to detect the same functions controlled by CtrA in different organisms also suggests that the prediction capability of CtrA-regulated genes is highly reliable.

The C. crescentus regulatory scheme of CtrA in other members of cluster A shows several variations, especially in Rhizobiales. For example, the control of CtrA on the 


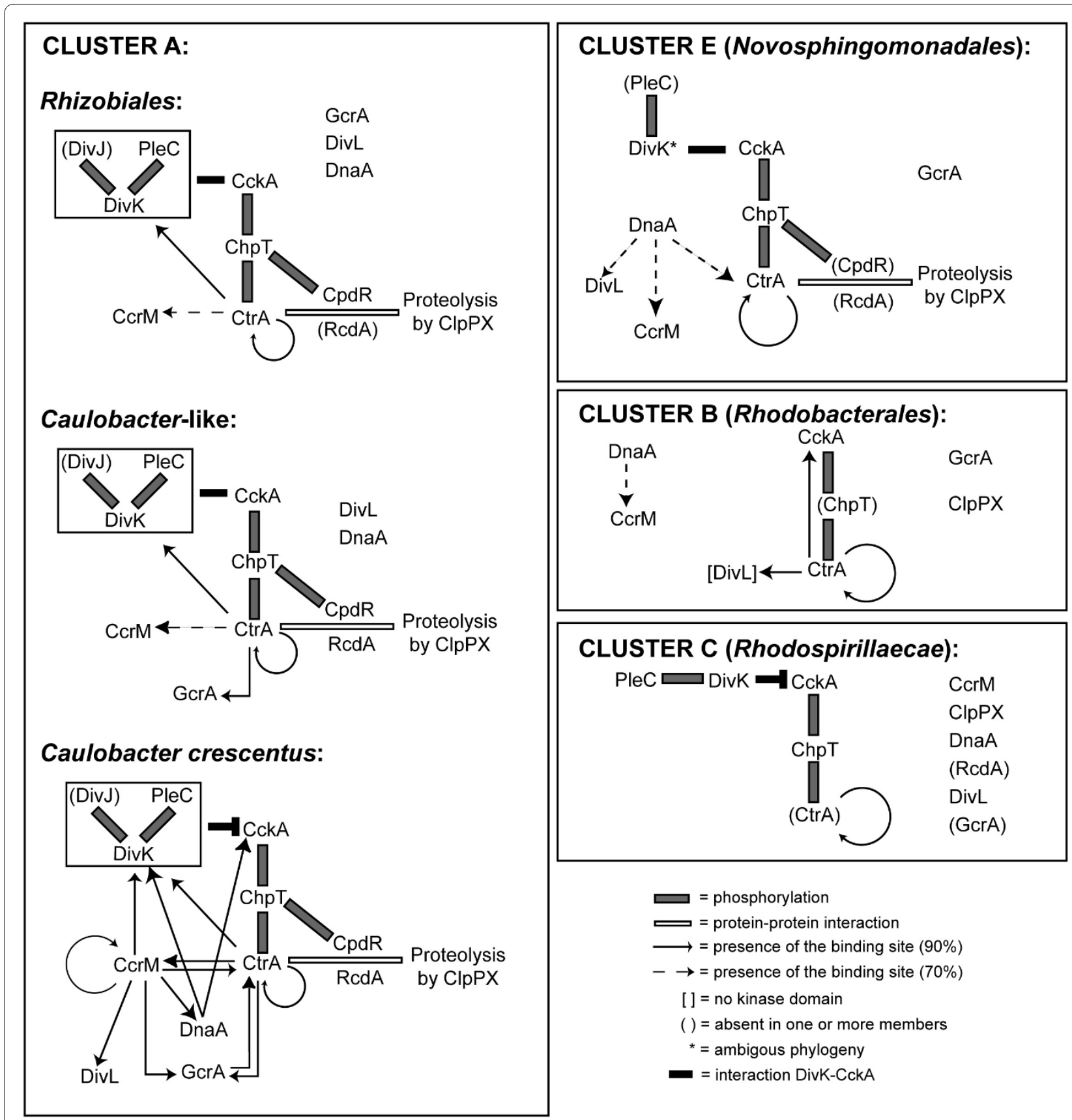

Figure 7 Regulatory circuits of clusters A, B, C, E. See the "Results" and "Discussion" sections for more details. Interactions via phosphorylation, as well as proteolysis, were suggested only considering the interaction demonstrated in Caulobacter. Moreover, DivK inhibition on CckA was considered, as in Biondi (2006), only in Caulobacter [20]. The presence of binding sites of transcription factors CtrA, DnaA and GcrA is shown as a continuous line if predicted binding sites were present in at least $90 \%$ of the gene promoters of a cluster. In contrast, the connection is shown as a dotted line for binding sites present in ca. 70\%. The Caulobacter-like group corresponds to B. japonicum, P. lavamentivorans and M. maris.

response regulator $\operatorname{div} K$, observed in C. crescentus, is shifted to the gene encoding the DivK kinase (divJ) and/ or the phosphatase $(p l e C)$ in most Rhizobiales. This observation may suggest that feedbacks can be conserved even when connections are rewired.

Another interesting feature revealed by this study is the control of CtrA on $c c k A$ and $\operatorname{div} L$ in several members of the Rhodobacterales (cluster B), which is coupled to the absence of the system regulating CckA activity (the DivK/ $\mathrm{Div} / \mathrm{PleC}$ two-component system). Thus, in cluster A members, CtrA controls the divK-divJ-pleC transcription and DivK presumably inhibits CckA activation that is indeed able to activate CtrA through a phosphorylation cascade, while in cluster $\mathrm{B}$ Rhodobacterales our results 
suggest that $\mathrm{Ctr} A$ acts directly on $c c k A$ transcription and thus it may directly modulate the CckA quantity in the cytoplasm.

\section{Other regulatory circuits (DivL-DivK and DnaA-GcrA-CtrA)}

Several conclusions can be made at the molecular level from this bioinformatic approach on specific factors such as DivL-DivK or the DnaA-GcrA-CtrA system. DivL, that still lacks a precise function, and DivK, have been connected in previous studies since DivL was detected in a yeast-two hybrid experiment using DivK as bait [55]. Alleles of $d i v L$ were also able to complement $\operatorname{divK}$ defective alleles $[24,56]$. A further association between DivL and DivK was found in our study whereby orthologs of DivL can be found in alpha-proteobacteria either as complete kinases (such as in Caulobacter) or without the kinase domain (data not shown); this latter form is always associated with the absence of DivK--suggesting that the kinase domain of DivL functions together with DivK. This observation is also consistent with previous studies that showed that DivL interacts with DivK using the kinase domain and has two separate functions in $\mathrm{Cau}$ lobacter [56]. The observation that in several alphas DivL keeps only the sensor part is also paralleled by previous studies showing that its kinase activity in Caulobacter is dispensable [56].

It can be concluded from this study that the DnaAGcrA-CtrA regulatory system in Caulobacter is not conserved throughout alpha-proteobacteria. It is however true that DnaA controls $g c r A$ in several members of cluster A (surprisingly this control in Caulobacter was not detected) and that GcrA controls ctrA in few alphas, including in Caulobacter. This observation might suggest that this important loop in the regulation of the Caulobacter cell cycle is instead dispensable in other bacteria of cluster A. This conclusion is also consistent with the result that regulation of ctrA transcription can be removed without affecting Caulobacter viability although robustness of the system might be compromised.

\section{Evolution of alpha-proteobacteria cell cycle}

Finally, the reconstruction of the evolution of the regulatory schemes found was undertaken following phylogeny proposed elsewhere [42,57]. Rickettsiales (clusters F and G) were excluded from this model due to their massive genome reduction that has been previously shown to be a consequence of the evolution of an obligate intracellular life in eukaryotic cells [58].

The regulation of cell cycle progression in Caulobacter has evolved in order to respond to a lifestyle in nutrientpoor environments but other alpha-proteobacteria occupy different ecological niches, suggesting that cell cycle regulation must respond to different requirements; from an evolutionary perspective this means that features found in Caulobacter should not be completely conserved in other alpha-proteobacteria, especially those experiencing different environments. Conversely, similarities between closer organisms were expected due to common phylogenetic ancestries.

Gupta and Mok [42] proposed that Rhodospirillales and Novosphingomonadales (clusters C, D and E) branched earlier than the other alphas (clusters A and B), and after Rickettsiales (clusters F and G). Two of those "older" clusters (C and E) show, in fact, a different arrangement of regulatory factors although none show circularity of the regulation created by feedbacks. From this primordial situation, when alphas were experimenting different options, two situations seem to have evolved: a complex circuit (cluster A) and a minimal organization (cluster B). In both clusters two interlaced oscillators are present: besides cluster $\mathrm{A}$ where this organization has already been proposed [20], cluster B, also shows a CtrA autoregulatory circuit and a CtrA-CckA-ChpT-CtrA oscillator. It is also interesting to point out that the minimal circuit of cluster $\mathrm{C}$ corresponds to a situation where $\mathrm{Ctr} A$ is not essential.

\section{Conclusions}

This is the first systematic attempt to translate the information accumulated over the years on cell cycle regulation in the model Caulobacter system into a common body of knowledge regarding the whole taxonomic group of alpha-proteobacteria. Results suggest that the scheme found in Caulobacter may work in closely related bacteria such as those belonging to Caulobacterales and Rhizobiales while in other alphas this conservation is lost even though several modules are present. Finally, this analysis represents an important step for all future cell cycle studies in alpha-proteobacteria, offering many experimental scenarios designed to confirm or reject the observations made here.

\section{Methods \\ Phylogenetic Tree}

To construct our reference phylogenetic tree, from the server of the RDP (ribosomal database project) [59], we downloaded the $E$. coli sequences corresponding to universal proteins in prokaryotes: FusA, IleS, LepA, LeuS, PyrG, RecA, RecG and RplB. Then we blasted these sequences against alpha-proteobacterial genomes chosen for analysis, retrieving the $\mathrm{BBH}$ for each protein in each genome. Each dataset was aligned using Muscle [60] and the obtained multi-alignments were then joined head-to-tail in a single concatamer of 5000 sites. This alignment was used with the software Mega 4 [61] running the Neighbor-Joining algorithm, 500 bootstrap replicates and the Dayhoff model of evolution.

\section{Ortholog Identification}

We used a dataset comprising all completely sequenced genomes of organisms belonging to the alpha-proteobac- 
teria for ortholog identification (Additional file 1, Table S1). Orthologous genes were identified with the so-called bidirectional best hit (BBH) criterion: the relation of gene $x$ in genome $A$ and gene $y$ in genome $B$ is called bidirectional best hit, when $x$ is the best hit of query $y$ against all genes in $A$ and vice versa. We used two different datasets of queries coming from C. crescentus and B. melitensis and we also performed a phylogenetic analysis of those proteins found using the $\mathrm{BBH}$ analysis; the Caulobacter dataset is derived from experimental data [13] while that from $B$. abortus is derived from both experimental [38] and bioinformatic analysis performed using Caulobacter genes. For multi-domain proteins we used separated domains and discarded highly variable regions from the analysis. The two sets of BBH sequences orthologous to Caulobacter and Brucella sequences were then combined to collect the maximum number of proteins. When $C$. crescentus and B.melitensis sequences retrieved two different proteins for the same gene, we collected both of them and used phylogenetic methods to solve the mismatch; when one of the two queries returned no $\mathrm{BBH}$ we used only the one available. We used a 0.0001 e-value threshold in the Blast analysis.

\section{Regulons of CtrA, GcrA, DnaA and CcrM methylation sites characterization}

Genes directly regulated by CtrA in alpha-proteobacteria genomes were identified using the following multilevel approach:

1) A Position Weight Matrix (PWM) describing CtrA binding sites in C. crescentus was obtained by using the AlignAce program [45] on upstream sequences of 54 genes previously identified as being part of the CtrA regulon [13]. We used such a matrix to scan all genomes considered in this work (see Additional File 1, Table S1 for all organisms list) with a sliding window approach and a scoring function from Schneider and collaborators (1986) [62]: $S_{i}=(1 / L) \Sigma_{j}[2+$ $\log _{2}\left(\mathrm{~F}_{\mathrm{ij}}\right)$ ], where $\mathrm{F}_{\mathrm{ij}}$ is the frequency of base $\mathrm{i}$ at position $j$ of the L-mer. This score, whose maximum for the best match using a CtrA position weight matrix is $\approx 1.22$, is a measure of the information content of a potential binding site.

2) We retained only motifs having a score $\geq 30 \%$ of the maximum score attainable with the given matrix and located in the range -100 to 400 nucleotides from the start codon of a gene.

3) Then we applied a Z-score transformation to highlight significant occurrences and take into account the background DNA implicitly: $\mathrm{Z}_{\mathrm{i}}=\left(\mathrm{S}_{\mathrm{i}}-\left\langle\mathrm{S}_{\mathrm{i}}\right\rangle\right) / \sigma_{\mathrm{i}}$, where $S_{\mathrm{i}}$ is calculated using the above formula, and $\left\langle\mathrm{S}_{\mathrm{i}}\right\rangle$ is the average score in an organism over all $\mathrm{L}$ mers and $\sigma_{i}$ is the corresponding standard deviation.
For GcrA and DnaA the strategy followed was the same, except that PWMs were obtained differently. For GcrA we obtained a PWM describing a motif enriched upstream of sequences controlled by GcrA as proposed by others [19]. By using microarray and GcrA deficient strains, a list of genes whose expression changes significantly when GcrA is mutated was obtained. We used the 48 genes with maximum fold change in that list. The corresponding upstream sequences were retrieved and analyzed with AlignAce [45] and MDscan [63]. In both cases a high scoring motif (16 bp, see Additional file 10, Figure S4) present in a high percentage of the input sequences was obtained, and was used for subsequent scanning of the genomes of our dataset (Figure 5 and Additional file 7, Table S4).

DnaA recognizes a sequence whose consensus in $\mathrm{Cau}$ lobacter is [TC] [TCG] [AG]TCCACA as previously reported [18]. In the same work, 15 DnaA binding sites were shown and were used to build a position weight matrix specific for DnaA (Additional file 10, Figure S4). However AlignAce and MDscan were not able to identify motifs for DnaA corresponding at least partially to the proposed consensus using two datasets of genes previously proposed [18]

Both GcrA and DnaA were then analyzed as reported in points 2 and 3 for CtrA. CcrM methylation targets were searched using a regular expression modelling the known target of this methyl-transferase (GANTC) [11].

\section{Functional Enrichment}

We took advantage of COG [64] categories to evaluate the functional enrichment of the genes identified with the approach described above. Supposing that the regulon dataset of a genome is composed of $\mathrm{N}$ genes, we counted the number of genes for each category and then repeated the same count for 10,000 N-sized groups of genes randomly picked from the genome. We obtain a p-value describing functional enrichment in the original dataset by counting how many times a COG category is more represented in the random group and then dividing it by the total number of repetitions performed.

\section{Additional material}

Additional file 1 Table S1. The list of the 65 organisms used in this work. Additional file 2 Detailed description of additional material. Legends of additional Tables and Figures.

Additional file $\mathbf{3}$ Figure S1. Phylogenetic profiling of BBH hits in the 65genome dataset

Additional file 4 Table S2. Bidirectional Best Hits.

Additional file $\mathbf{5}$ Figure S2. Phylogenetic trees of cell cycle related proteins.

Additional file 6 Table S3. CtrA, DnaA, GcrA and CcrM binding sites.

Additional file 7 Table S4. Numerical values for Figures 3, 5 and S3.

Additional file $\mathbf{8}$ Table S5. Numerical values for Figure 4.

Additional file 9 Figure S3. Control of CcrM on cell cycle genes. 
Additional file 10 Figure S4. Sequence logo of the putative GcrA motif.

\section{Abbreviations}

PWM: position weight matrix; $\mathrm{BBH}$ : bidirectional best hit; COG: clusters of orthologous groups of proteins; SMART: simple modular architecture research tool

\section{Authors' contributions}

EGB planned the project; MB performed the analysis; MF, RF, AM, LF and MB helped with the analytical work and the writing process. $E G B$ and $M B$ wrote the manuscript and created Tables and Figures. All authors read and approved the final version of the manuscript.

\section{Acknowledgements}

We thank Prof. Michael Laub for critical reading of the manuscript. We thank also Mary C. Forrest for editing. Emanuele G. Biondi is supported by the "Fondazione Adriano Buzzati-Traverso" and "Ente Cassa di Risparmio di Firenze".

\section{Author Details}

'Department of Evolutionary Biology, University of Florence, via Romana, 17, Florence, Italy and 2Laboratoire de Biométrie et Biologie Evolutive, UMR CNRS 5558, Université Lyon 1, 43, bvd du 11 novembre, Lyon, France

Received: 27 November 2009 Accepted: 28 April 2010 Published: 28 April 2010

\section{References}

1. Fawcett $P$, Eichenberger $P$, Losick R, Youngman $P$ : The transcriptional profile of early to middle sporulation in Bacillus subtilis. Proc Natl Acad Sci USA 2000, 97:8063-8068.

2. Haeusser DP, Levin PA: The great divide: coordinating cell cycle events during bacterial growth and division. Curr Opin Microbiol 2008, 11:94-99.

3. Bowers LM, Shapland EB, Ryan KR: Who's in charge here? Regulating cell cycle regulators. Curr Opin Microbiol 2008, 11:547-552.

4. Laub MT, Shapiro L, McAdams HH: Systems biology of Caulobacter. Annu Rev Genet 2007, 41:429-441.

5. Purcell EB, Boutte CC, Crosson S: Two-component signaling systems and cell cycle control in Caulobacter crescentus. Adv Exp Med Biol 2008, 631:122-130.

6. Skerker JM, Laub MT: Cell-cycle progression and the generation of asymmetry in Caulobacter crescentus. Nat Rev Microbiol 2004 2:325-337.

7. Domian IJ, Quon KC, Shapiro L: Cell type-specific phosphorylation and proteolysis of a transcriptional regulator controls the G1-to-S transition in a bacterial cell cycle. Cell 1997, 90:415-424.

8. Quon KC, Marczynski GT, Shapiro L: Cell cycle control by an essential bacterial two-component signal transduction protein. Cell 1996, 84:83-93.

9. Reisenauer A, Quon K, Shapiro L: The CtrA response regulator mediates temporal control of gene expression during the Caulobacter cell cycle. J Bacteriol 1999, 181:2430-2439.

10. Biondi EG, Skerker JM, Arif M, Prasol MS, Perchuk BS, Laub MT: A phosphorelay system controls stalk biogenesis during cell cycle progression in Caulobacter crescentus. Mol Microbiol 2006, 59:386-401.

11. Collier J, McAdams HH, Shapiro L: A DNA methylation ratchet governs progression through a bacterial cell cycle. Proc Natl Acad Sci USA 2007, 104:17111-17116.

12. Jones SE, Ferguson NL, Alley MR: New members of the ctrA regulon: the major chemotaxis operon in Caulobacter is CtrA dependent. Microbiology 2001, 147:949-958.

13. Laub MT, Chen SL, Shapiro L, McAdams HH: Genes directly controlled by CtrA, a master regulator of the Caulobacter cell cycle. Proc Natl Acad Sci USA 2002, 99:4632-4637

14. Skerker JM, Shapiro L: Identification and cell cycle control of a novel pilus system in Caulobacter crescentus. EMBO J 2000, 19:3223-3234.

15. Wortinger M, Sackett MJ, Brun YV: CtrA mediates a DNA replication checkpoint that prevents cell division in Caulobacter crescentus. EMBO J 2000, 19:4503-4512.
16. Collier J, Murray SR, Shapiro L: DnaA couples DNA replication and the expression of two cell cycle master regulators. EMBO J 2006, 25:346-356.

17. Gorbatyuk B, Marczynski GT: Regulated degradation of chromosome replication proteins DnaA and CtrA in Caulobacter crescentus. Mol Microbiol 2005, 55:1233-1245.

18. Hottes AK, Shapiro L, McAdams HH: DnaA coordinates replication initiation and cell cycle transcription in Caulobacter crescentus. Mol Microbiol 2005, 58:1340-1353.

19. Holtzendorff J, Hung D, Brende P, Reisenauer A, Viollier PH, McAdams HH, Shapiro L: Oscillating global regulators control the genetic circuit driving a bacterial cell cycle. Science 2004, 304:983-987.

20. Biondi EG, Reisinger SJ, Skerker JM, Arif M, Perchuk BS, Ryan KR, Laub MT: Regulation of the bacterial cell cycle by an integrated genetic circuit. Nature 2006, 444:899-904.

21. Jacobs C, Domian IJ, Maddock JR, Shapiro L: Cell cycle-dependent polar localization of an essential bacterial histidine kinase that controls DNA replication and cell division. Cell 1999, 97:111-120.

22. Burton GJ, Hecht GB, Newton A: Roles of the histidine protein kinase pleC in Caulobacter crescentus motility and chemotaxis. J Bacteriol 1997, 179:5849-5853.

23. Ohta N, Lane T, Ninfa EG, Sommer JM, Newton A: A histidine protein kinase homologue required for regulation of bacterial cell division and differentiation. Proc Natl Acad Sci USA 1992, 89:10297-10301.

24. Sommer JM, Newton A: Pseudoreversion analysis indicates a direct role of cell division genes in polar morphogenesis and differentiation in Caulobacter crescentus. Genetics 1991, 129:623-630.

25. Wu J, Ohta N, Newton A: An essential, multicomponent signal transduction pathway required for cell cycle regulation in Caulobacter. Proc Natl Acad Sci USA 1998, 95:1443-1448.

26. Weiss $V$, Kramer G, Dunnebier T, Flotho A: Mechanism of regulation of the bifunctional histidine kinase NtrB in Escherichia coli. J Mol Microbiol Biotechnol 2002, 4:229-233.

27. Jacobs C, Hung D, Shapiro L: Dynamic localization of a cytoplasmic signal transduction response regulator controls morphogenesis during the Caulobacter cell cycle. Proc Natl Acad Sci USA 2001 98:4095-4100

28. Wheeler RT, Shapiro L: Differential localization of two histidine kinases controlling bacterial cell differentiation. Mol Cell 1999, 4:683-694.

29. Radhakrishnan SK, Thanbichler M, Viollier PH: The dynamic interplay between a cell fate determinant and a lysozyme homolog drives the asymmetric division cycle of Caulobacter crescentus. Genes Dev 2008 22:212-225

30. Iniesta AA, McGrath PT, Reisenauer A, McAdams HH, Shapiro L: A phospho-signaling pathway controls the localization and activity of a protease complex critical for bacterial cell cycle progression. Proc Natl Acad SciUSA 2006, 103:10935-10940.

31. Jenal U, Fuchs T: An essential protease involved in bacterial cell-cycle control. EMBO J 1998, 17:5658-5669.

32. McGrath PT, Iniesta AA, Ryan KR, Shapiro L, McAdams HH: A dynamically localized protease complex and a polar specificity factor control a cell cycle master regulator. Cell 2006, 124:535-547.

33. Ryan KR: Partners in crime: phosphotransfer profiling identifies a multicomponent phosphorelay. Mol Microbiol 2006, 59:361-363.

34. Ryan KR, Huntwork S, Shapiro L: Recruitment of a cytoplasmic response regulator to the cell pole is linked to its cell cycle-regulated proteolysis. Proc Natl Acad Sci USA 2004, 101:7415-7420.

35. Hallez R, Bellefontaine AF, Letesson JJ, De Bolle X: Morphological and functional asymmetry in alpha-proteobacteria. Trends Microbiol 2004, 12:361-365

36. Lang AS, Beatty JT: Genetic analysis of a bacterial genetic exchange element: the gene transfer agent of Rhodobacter capsulatus. Proc Natl Acad Sci USA 2000, 97:859-864.

37. Lang AS, Beatty JT: A bacterial signal transduction system controls genetic exchange and motility. J Bacteriol 2002, 184:913-918.

38. Bellefontaine AF, Pierreux CE, Mertens P, Vandenhaute J, Letesson JJ, De Bolle $X$ : Plasticity of a transcriptional regulation network among alphaproteobacteria is supported by the identification of CtrA targets in Brucella abortus. Mol Microbiol 2002, 43:945-960.

39. Robertson GT, Reisenauer A, Wright R, Jensen RB, Jensen A, Shapiro L, Roop RM: The Brucella abortus CcrM DNA methyltransferase is 
essential for viability, and its overexpression attenuates intracellular replication in murine macrophages. J Bacterio/ 2000, 182:3482-3489.

40. Miller TR, Belas R: Motility is involved in Silicibacter sp. TM1040 interaction with dinoflagellates. Environ Microbiol 2006, 8:1648-1659.

41. Kahng LS, Shapiro L: The CcrM DNA methyltransferase of Agrobacterium tumefaciens is essential, and its activity is cell cycle regulated. J Bacteriol 2001, 183:3065-3075.

42. Gupta RS, Mok A: Phylogenomics and signature proteins for the alpha proteobacteria and its main groups. BMC Microbiol 2007, 7:106.

43. Pellegrini M, Marcotte EM, Thompson MJ, Eisenberg D, Yeates TO: Assigning protein functions by comparative genome analysis: protein phylogenetic profiles. Proc Natl Acad Sci USA 1999, 96:4285-4288.

44. Laub MT, McAdams HH, Feldblyum T, Fraser CM, Shapiro L: Global analysis of the genetic network controlling a bacterial cell cycle. Science 2000, 290:2144-2148.

45. Roth FP, Hughes JD, Estep PW, Church GM: Finding DNA regulatory motifs within unaligned noncoding sequences clustered by wholegenome mRNA quantitation. Nat Biotechnol 1998, 16:939-945.

46. Brassinga AK, Siam R, McSween W, Winkler H, Wood D, Marczynski GT: Conserved response regulator $\mathrm{Ctr} A$ and IHF binding sites in the alphaproteobacteria Caulobacter crescentus and Rickettsia prowazekii chromosomal replication origins. J Bacteriol 2002, 184:5789-5799.

47. Stephens C, Reisenauer A, Wright R, Shapiro L: A cell cycle-regulated bacterial DNA methyltransferase is essential for viability. Proc Nat/ Acad Sci USA 1996, 93:1210-1214

48. Wright R, Stephens C, Shapiro L: The CcrM DNA methyltransferase is widespread in the alpha subdivision of proteobacteria, and its essential functions are conserved in Rhizobium meliloti and Caulobacter crescentus. J Bacteriol 1997, 179:5869-5877.

49. Hansen FG, Christensen BB, Atlung T: Sequence characteristics required for cooperative binding and efficient in vivo titration of the replication initiator protein DnaA in E. coli. J Mol Biol 2007, 367:942-952.

50. Ishikawa S, Ogura Y, Yoshimura M, Okumura H, Cho E, Kawai Y, Kurokawa K, Oshima T, Ogasawara N: Distribution of stable DnaA-binding sites on the Bacillus subtilis genome detected using a modified ChIP-chip method. DNA Res 2007, 14:155-168.

51. Collier J, Shapiro L: Spatial complexity and control of a bacterial cell cycle. Curr Opin Biotechnol 2007, 18:333-340.

52. Barnett MJ, Hung DY, Reisenauer A, Shapiro L, Long SR: A homolog of the CtrA cell cycle regulator is present and essential in Sinorhizobium meliloti. J Bacteriol 2001, 183:3204-3210.

53. McGrath PT, Lee H, Zhang L, Iniesta AA, Hottes AK, Tan MH, Hillson NJ, Hu P, Shapiro L, McAdams HH: High-throughput identification of transcription start sites, conserved promoter motifs and predicted regulons. Nat Biotechnol 2007, 25:584-592.

54. Sibley CD, MacLellan SR, Finan T: The Sinorhizobium meliloti chromosomal origin of replication. Microbiology 2006, 152:443-455.

55. Ohta N, Newton A: The core dimerization domains of histidine kinases contain recognition specificity for the cognate response regulator. $J$ Bacterio/ 2003, 185:4424-4431.

56. Reisinger SJ, Huntwork S, Viollier PH, Ryan KR: DivL performs critical cell cycle functions in Caulobacter crescentus independent of kinase activity. J Bacterio/ 2007, 189:8308-8320

57. Gupta RS: Protein signatures distinctive of alpha proteobacteria and its subgroups and a model for alpha-proteobacterial evolution. Crit Rev Microbiol 2005, 31:101-135.

58. Sallstrom B, Andersson SG: Genome reduction in the alphaProteobacteria. Curr Opin Microbiol 2005, 8:579-585.

59. Cole JR, Wang Q, Cardenas E, Fish J, Chai B, Farris RJ, Kulam-SyedMohideen AS, McGarrell DM, Marsh T, Garrity GM, Tiedje JM: The Ribosomal Database Project: improved alignments and new tools for rRNA analysis. Nucleic Acids Res 2009, 37:D141-145.

60. Edgar RC: MUSCLE: a multiple sequence alignment method with reduced time and space complexity. BMC Bioinformatics 2004, 5:113.

61. Tamura K, Dudley J, Nei M, Kumar S: MEGA4: Molecular Evolutionary Genetics Analysis (MEGA) software version 4.0. Mol Biol Evol 2007, 24:1596-1599.

62. Schneider TD, Stormo GD, Gold L, Ehrenfeucht A: Information content of binding sites on nucleotide sequences. J Mol Biol 1986, 188:415-431.

63. Liu XS, Brutlag DL, Liu JS: An algorithm for finding protein-DNA binding sites with applications to chromatin-immunoprecipitation microarray experiments. Nat Biotechnol 2002, 20:835-839.
64. Tatusov RL, Natale DA, Garkavtsev IV, Tatusova TA, Shankavaram UT, Rao BS, Kiryutin B, Galperin MY, Fedorova ND, Koonin EV: The COG database: new developments in phylogenetic classification of proteins from complete genomes. Nucleic Acids Res 2001, 29:22-28.

65. Whelan S, Lio P, Goldman N: Molecular phylogenetics: state-of-the-art methods for looking into the past. Trends Genet 2001, 17:262-272.

doi: 10.1186/1752-0509-4-52

Cite this article as: Brilli et al., The diversity and evolution of cell cycle regulation in alpha-proteobacteria: a comparative genomic analysis BMC Systems Biology 2010, 4:52

\section{Submit your next manuscript to BioMed Central and take full advantage of:}

- Convenient online submission

- Thorough peer review

- No space constraints or color figure charges

- Immediate publication on acceptance

- Inclusion in PubMed, CAS, Scopus and Google Scholar

- Research which is freely available for redistribution 